\title{
PHONETIC ORTHOGRAPHY USED IN THIS BOOK
}

Throughout this book, words and elements cited from Indian languages are written in italics in the normalized phonetic orthography that is detailed in Table 1 (for other phonetic orthographies that have been used in transcribing California languages, see Appendix C). When names and occasionally other words are cited in their traditional spelling, these forms are not italicized and are sometimes enclosed in double quotes.

Here are a few additional conventions:

ๆ indicates a cross-reference to another section of this book

[ ] (enclosing an Indian form) indicates a phonetic transcription

* (preceding an Indian form) indicates that it is reconstructed or hypothetical

TABLE $1 \mathrm{~A}$

Consonants

\begin{tabular}{|c|c|c|c|c|c|c|c|c|c|c|}
\hline & Bilabial & $\begin{array}{l}\text { Inter- } \\
\text { dental }\end{array}$ & $\begin{array}{l}\text { Dental- } \\
\text { Alveolar }\end{array}$ & $\begin{array}{l}\text { Retro- } \\
\text { flex }\end{array}$ & Lateral & Palatal & Velar & $\begin{array}{c}\text { Labio- } \\
\text { velar }\end{array}$ & $\begin{array}{l}\text { Post- } \\
\text { velar }\end{array}$ & Laryngeal \\
\hline \multicolumn{11}{|l|}{ Stops } \\
\hline Plain & $\mathrm{p}$ & & $\mathrm{t}$ & ț & & & $\mathrm{k}$ & $\mathrm{k}^{\mathrm{w}}$ & $\mathrm{q}$ & $?$ \\
\hline Aspirated & $\mathrm{p}^{\mathrm{h}}$ & & $t^{\mathrm{h}}$ & $\underline{t}^{\mathrm{h}}$ & & & $\mathrm{k}^{\mathrm{h}}$ & $\mathrm{k}^{\mathrm{hw}}$ & $\mathrm{q}^{\mathrm{h}}$ & \\
\hline Glottalized & p' & & $t^{\prime}$ & $t^{\prime}$ & & & $\mathrm{k}^{\prime}$ & $\mathrm{k}^{\prime \mathrm{w}}$ & q' & \\
\hline Voiced & $\mathrm{b}$ & & d & da & & & $\mathrm{g}$ & $g^{w}$ & $\dot{\mathrm{g}}$ & \\
\hline \multicolumn{11}{|l|}{ Fricatives } \\
\hline Plain & $\phi, \mathrm{f}$ & $\theta$ & $\mathrm{s}$ & $s$ & t & š & $\mathrm{x}$ & $\mathrm{x}^{\mathrm{w}}$ & $x$ & $\mathrm{~h}$ \\
\hline Voiced & $\beta, \mathrm{v}$ & ð & $\mathrm{z}$ & $\mathrm{z}$ & & $\check{z}$ & $\gamma$ & $\gamma^{\mathrm{w}}$ & $\gamma$ & \\
\hline \multicolumn{11}{|l|}{ Affricates } \\
\hline Plain & & & $\mathrm{c}$ & ç & $x$ & $\check{c}$ & & & & \\
\hline Aspirated & & & $c^{\mathrm{h}}$ & $c^{h}$ & $x^{\mathrm{h}}$ & $\check{c}^{\mathrm{h}}$ & & & & \\
\hline Glottalized & & & $c^{\prime}$ & ç' & $x^{\prime}$ & с̌' & & & & \\
\hline Voiced & & & 3 & & & $\check{3}$ & & & & \\
\hline
\end{tabular}

Nasals

$\begin{array}{llllll}\text { Voiced } & \mathrm{m} & \mathrm{n} & \mathrm{n}^{\mathrm{y}} & \mathrm{j} & \mathrm{j}^{\mathrm{w}} \\ \text { Voiceless } & \mathrm{M} & \mathrm{N} & & \mathrm{\eta} & \end{array}$

(continued) 


\begin{tabular}{|c|c|c|c|c|c|c|c|c|c|c|}
\hline & Bilabial & $\begin{array}{l}\text { Inter- } \\
\text { dental }\end{array}$ & $\begin{array}{l}\text { Dental- } \\
\text { Alveolar }\end{array}$ & $\begin{array}{l}\text { Retro- } \\
\text { flex }\end{array}$ & Lateral & Palatal & Velar & $\begin{array}{l}\text { Labio- } \\
\text { velar }\end{array}$ & $\begin{array}{l}\text { Post- } \\
\text { velar }\end{array}$ & Laryngeal \\
\hline \multicolumn{11}{|c|}{ Approximants } \\
\hline Voiced & $\mathrm{w}$ & & & $\mathrm{r}$ & 1 & $\mathrm{y}$ & & & & \\
\hline Voiceless & $\mathrm{W}$ & & & $\mathrm{R}$ & $\mathrm{L}$ & $\mathrm{Y}$ & & & & \\
\hline
\end{tabular}

\section{Palatalization}

$\mathrm{k}^{\mathrm{y}}$ or $\mathrm{k}$ (velars), $\mathrm{l}^{\mathrm{y}}$ (laterals), $\mathrm{t}^{\mathrm{y}} \mathrm{n}^{\mathrm{y}} \mathrm{s}^{\mathrm{y}}$ (dental-alveolars).*

Trills and taps

$\tilde{\mathrm{r}}$ (trill), $\mathrm{c}$ (tap or flap).

Prenasalized voiced stops

${ }^{\mathrm{n}} \mathrm{d}$, etc.

Glottalized nasals and approximants

'm, etc. (glottal stop released as nasal or approximant segment), m' (nasal or approximant segment closed by glottal stop), m (nasal or approximant segment with simultaneous glottal stricture). Where there is allophonic variation or the exact phonetic representation of the phoneme is not clear, m, etc. is used as a cover symbol except for glottalized laterals, where for typographic reasons l' is used.

Gemination (contrastive consonant length)

marked by doubling the letter (patta).

Rare consonants

$\mathrm{s}^{\mathrm{h}}$ (aspirated/fortis voiceless fricative, occurs in Central Chumash); $\hbar$ (voiceless pharyngeal fricative, occurs in Achumawi); kx (velar affricate, reconstituted Esselen phoneme).

*A y superscript is used systematically by linguists working with Yuman languages to mark the palatalized nasals $\left(n^{y}\right)$, laterals $\left(l^{y}\right)$, and velar stops $\left(k^{y}\right)$ that are phonemically distinct in many of those languages. This usage is generalized in this book except in California Athabaskan, where an alternative symbol with a subscript semicircle $(k)$ is employed for the palatalized velar stop in order to avoid awkward double superscripts where aspiration or glottalization must also be marked.

TABLE 1 B

Vowels

\begin{tabular}{|c|c|c|c|c|}
\hline & \multicolumn{2}{|c|}{ Front } & \multicolumn{2}{|c|}{ Nonfront/Back } \\
\hline & Unrounded & Rounded & Unrounded & Rounded \\
\hline High & $\mathrm{i}$ & $\ddot{\mathrm{u}}$ & $\ddot{1}$ & $\mathrm{u}$ \\
\hline Lower-high & I & & & $\mathrm{U}$ \\
\hline Higher-mid & $\mathrm{e}$ & $\ddot{0}$ & $\ddot{\mathrm{e}}$ & o \\
\hline Lower-mid & $\varepsilon$ & $\ddot{\jmath}$ & ə & כ \\
\hline Higher-low & æ & & $\Lambda$ & \\
\hline Low & $\mathrm{a}$ & & $\mathrm{a}$ & $\mathrm{D}$ \\
\hline
\end{tabular}

Contrastive vowel length

marked by a colon (a:, e:)

Nasalization

marked by a superscript tilde; occurs in the California region only in Northern Yukian, where it is a secondary feature of the reduced central vowel (ə) [ว̃].

R-color

marked by superscript $r$ in Serrano-Vanyume $\left(a^{r}, e^{r}\right)$; the $r$-colored central vowel $\left[\partial^{r}\right]$ that occurs in Yurok is written as $r$ with an underdot $(r)$. 